\title{
Wide and Fast Wavelength-Swept Fiber Laser Based on Dispersion Tuning for Dynamic Sensing
}

\author{
Shinji Yamashita, Yuichi Nakazaki, Ryosei Konishi, and Osamu Kusakari \\ Department of Electrical Engineering and Information Systems, The University of Tokyo, 7-3-1 Hongo, Bunkyo-ku, \\ Tokyo 113-8656, Japan \\ Correspondence should be addressed to Shinji Yamashita, syama@ee.t.u-tokyo.ac.jp
}

Received 13 February 2009; Accepted 12 June 2009

Recommended by Christos Riziotis

We have developed a unique wide and fast wavelength-swept fiber laser for dynamic and accurate fiber sensing. The wavelength tuning is based on the dispersion tuning technique, which simply modulates the loss/gain in the dispersive laser cavity. By using wideband semiconductor optical amplifiers (SOAs), the sweep range could be as wide as $\sim 180 \mathrm{~nm}$. Since the cavity contains no mechanical components, such as tunable filters, we could achieve very high sweep rate, as high as $\sim 200 \mathrm{kHz}$. We have realized the swept lasers at three wavelength bands, $1550 \mathrm{~nm}, 1300 \mathrm{~nm}$, and $800 \mathrm{~nm}$, using SOAs along with erbium-doped fiber amplifiers (EDFAs), and in two laser configurations, ring and linear ones. We also succeeded in applying the swept laser for a dynamic fiberBragg grating (FBG) sensor system. In this paper, we review our researches on the wide and fast wavelength-swept fiber lasers.

Copyright ( $) 2009$ Shinji Yamashita et al. This is an open access article distributed under the Creative Commons Attribution License, which permits unrestricted use, distribution, and reproduction in any medium, provided the original work is properly cited.

\section{Introduction}

Wavelength-tunable lasers are versatile both in telecom and sensing applications. For the sensing applications, wide tuning range is needed to improve the spatial resolution and/or measurement range, and fast tuning (sweep) is required to enhance the measurement speed for dynamic sensing. Figure 1 shows two examples of wavelength-swept laser-based sensing systems, fiber Bragg grating (FBG) sensor system (Figure 1(a)) [1] and optical coherence tomography (OCT) system (Figure 1(b)) [2]. In the FBG sensor system, the strain or temperature change is decoded as the shift of the Bragg wavelength of the FBG in the position. Since multipoint sensing is achieved by multiplexing FBGs in wavelength, the tuning range determines the number of FBGs $(\propto$ measurement range). In case of dynamic FBG sensing, such as vibrations, the tuning speed has to be faster than the vibration. For the FBG sensor system, $1550 \mathrm{~nm}$ wavelength region is mostly used because the fiber loss is minimum and telecom components are readily available. In the OCT system, scattered and reflected light from the object under test, typically a living tissue, is resolved in the frequency domain by interference with the reference light, whose spatial resolution is inversely proportional to the tuning range. For the three-dimensional (3D) OCT measurement, the laser beam has to be deflected and scanned spatially. Thus, the dynamic and high-resolution 3D-OCT measurement requires extremely wide tuning range over $100 \mathrm{~nm}$, and extremely fast sweep rate over $100 \mathrm{kHz}$. For the retinal OCT, $1300 \mathrm{~nm}$ wavelength region is mostly chosen in order to compromise the scattering (strong at shorter wavelength) and water absorption (large at longer wavelength). It should be noted that such swept lasers do not have to be CW ones, but can be the pulsed ones as long as the interference is assured.

Many kinds of wavelength-swept lasers, either laser diode- (LD-) based or fiber laser-based, have been proposed so far. External cavity tunable LDs are commercially available at several wavelength bands and has wide tuning range over $100 \mathrm{~nm}$ [3], but the tuning speed is not fast because the tuning is achieved by the rotation of the bulk grating. The most widely used wavelength-swept lasers consist of a wide gain medium, and a fast tunable optical filter, as illustrated in Figure 2(a). The wide gain medium is typically a semiconductor optical amplifier (SOA) or an erbiumdoped fiber amplifier (EDFA). EDFAs can output high 


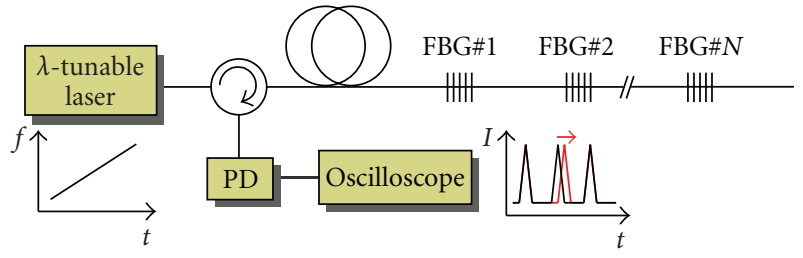

(a)

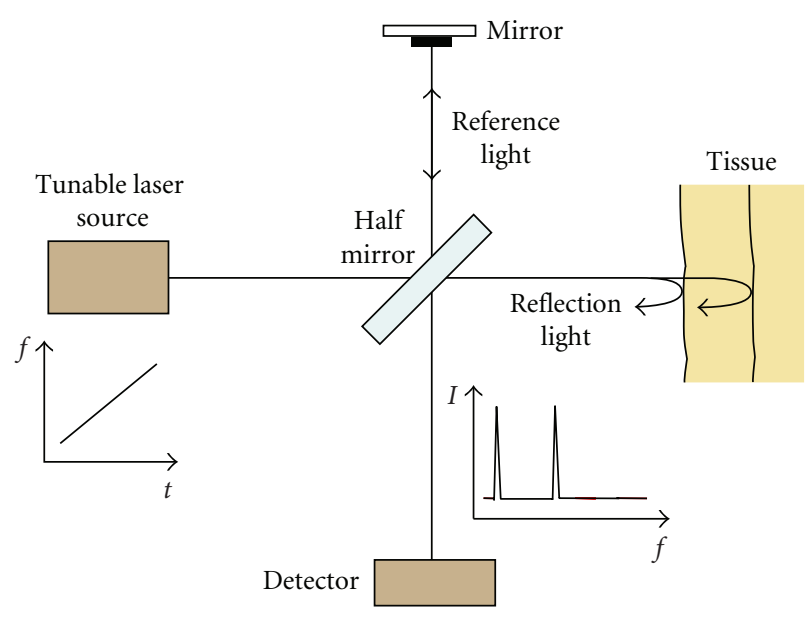

(b)

FIGURE 1: Wavelength-swept laser-based sensing systems (a) FBG sensor system (b) OCT system.

power and EDFA-based lasers can be tunable over $80 \mathrm{~nm}$ [4], but its wavelength band is limited to $1550 \mathrm{~nm}$. So the more preferred gain medium for sensing applications is the SOA, which can work at many wavelength bands $(1550 \mathrm{~nm}, 1300 \mathrm{~nm}, 1000 \mathrm{~nm}$, and $800 \mathrm{~nm})$ with wide gain bandwidth over $100 \mathrm{~nm}$. As for sweep speed, there are two limiting factors, one is the sweep speed of the optical filters, and the other is the photon lifetime in the laser cavity, which is inversely proportional to the cavity length. There have been several fast tunable optical filters, such as piezo-transducer- (PZT-) based tunable Fabry-Perot filters (FFP) [5], and polygonal mirror scanners [6]. They are basically mechanically tunable filters, in which the sweep speed is normally limited below a few tens of $\mathrm{kHz}$ by the mechanical movements. FFPs have been shown to be able to be swept much faster, a few hundreds of $\mathrm{kHz}$, by utilizing the resonance of specially-designed PZT [7], but the endurance of the PZT in the resonant mode is not clear. In terms of the second limiting factor, the cavity length, the SOA-based lasers are more advantageous than the EDFA-based lasers since the cavity can be shorter. As a different approach to this issue, so-called Fourier-domain mode locking (FDML) has been proposed recently, in which the sweep time is set to be equal to one round trip time of the cavity [7]. With the FDML, $105 \mathrm{~nm}$ tuning range and $290 \mathrm{kHz}$ sweep rate have been achieved, but the endurance of the FFP in such high speed is still in question.

In this review paper, we present our proposed novel and different type of wide and fast wavelength-tunable fiber laser

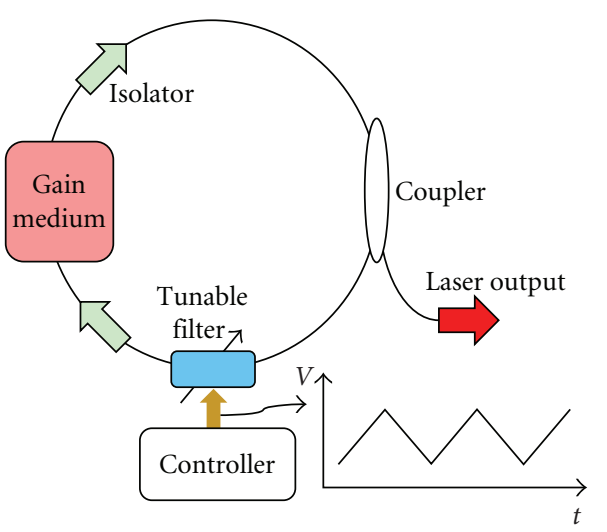

(a)

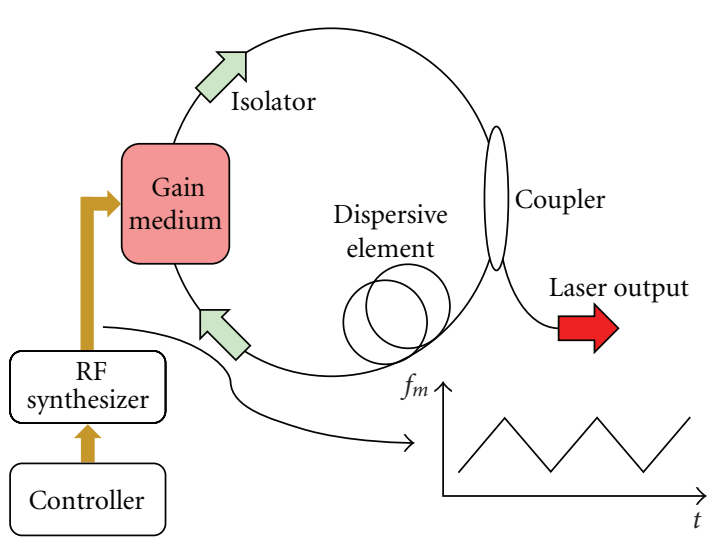

(b)

FIGURE 2: Wavelength-swept fiber lasers: (a) tunable filter-based laser (b) dispersion-tuned laser.

[8]. It uses the same gain medium with the conventional swept fiber lasers, but does not use any tunable filters. The tuning mechanism of our swept laser is so-called "dispersion tuning" technique, which simply modulates the loss/gain in the dispersive laser cavity, as illustrated in Figure 2(b). By using wideband SOAs, the sweep range could be as large as $\sim 180 \mathrm{~nm}$. Since the cavity contains no mechanical components, such as tunable filters, we could achieve very high sweep rate, as high as $\sim 200 \mathrm{kHz}$. We have realized the swept lasers at three wavelength bands, $1550 \mathrm{~nm}, 1300 \mathrm{~nm}$, and $800 \mathrm{~nm}$, using SOAs along with EDFAs, and in two laser configurations, ring and linear ones. We also succeeded in applying the swept laser for dynamic FBG sensors. We review our researches on the wide and fast wavelength-swept fiber lasers.

\section{Principle of Dispersion Tuning}

The laser cavity has discrete longitudinal resonance modes. The resonance condition of the ring cavity is expressed as

$$
\beta\left(\omega_{K}\right) L=2 K \pi,
$$

where $K$ is an integer expressing the mode number, $\beta(\omega)$ denotes the propagation constant at an angular frequency 
$\omega, \omega_{K}$ is the angular frequency of the $K$ th resonance mode, and $L$ is the cavity length. However, (1) can be transformed to

$$
\left(\beta\left(\omega_{K+1}\right)-\beta\left(\omega_{K}\right)\right) L=2 \pi .
$$

Spacing between adjacent modes or the free-spectral range (FSR) $F$ of the laser cavity is expressed, using the relation $\beta=$ $n \omega / c$ as

$$
F=\frac{\omega_{K+1}-\omega_{K}}{2 \pi}=\frac{c}{n L},
$$

where $n$ is the effective refractive index in the cavity, and $c$ is the speed of light in the vacuum. Note that $n$ is assumed to be a constant in deriving (3).

In the fiber laser cavity, $L$ is long, thus the spacing between adjacent modes is small. Here we assume $\omega_{K+1}-$ $\omega_{K} \ll \omega_{K}$, then (2) becomes

$$
\left(\omega_{K+1}-\omega_{K}\right) \frac{d \beta}{d \omega} L=2 \pi .
$$

However, (4) leads to another but more general expression of FSR as

$$
F=\left[\frac{d \beta}{d \omega} L\right]^{-1}
$$

We can lock the lasing modes and generate a short pulse train by applying the modulation at a frequency, which is well known as the active mode locking technique [9]. The modulation frequency $f_{m}$ to the cavity has to match with an integer $(N)$ times of the FSR $(=N \times F)$, where $N$ is the order of harmonics (harmonic mode locking). Without the chromatic dispersion in the cavity, $F$ is a constant over entire frequency. In this case, the lasing occurs at the gain peak wavelength, regardless whether the modes are locked or not.

When the chromatic dispersion exists in the laser cavity, $F$ becomes dependent on the frequency, $F(\omega)$. By expanding $\beta(\omega)$ around a frequency $\omega_{0}$ with the Taylor series, (5) becomes

$$
\begin{aligned}
F(\omega) & =\frac{1}{L}\left[\beta_{1}+\beta_{2}\left(\omega-\omega_{0}\right)+\frac{1}{2} \beta_{3}\left(\omega-\omega_{0}\right)^{2}+\cdots\right]^{-1} \\
& \approx F_{0}\left[1-\frac{\beta_{2}}{\beta_{1}} \Delta \omega-\frac{1}{2} \frac{\beta_{3}}{\beta_{1}} \Delta \omega^{2}-\cdots\right],
\end{aligned}
$$

where $F_{0}=1 /\left(\beta_{1} L\right)$, which is the initial FSR at $\omega=\omega_{0}$, and $\Delta \omega=\omega-\omega_{0}$. Here we assumed that terms containing $\Delta \omega$ are much smaller than the first term. Neglecting the higher order chromatic dispersion $\left(\beta_{3}, \beta_{4}, \ldots\right)$, the change of the FSR from $F_{0}$ by the change of frequency, $\Delta F(\omega)$, is expressed as

$$
\Delta F(\omega)=-F_{0} \frac{\beta_{2}}{\beta_{1}} \Delta \omega .
$$

However, (7) implies that the FSR is enlarged or reduced (depending on the sign of $\beta_{2}$ ) linearly in proportion to the change of frequency, as shown in Figure 3. The dispersion tuning is a technique using the unevenly spaced resonance

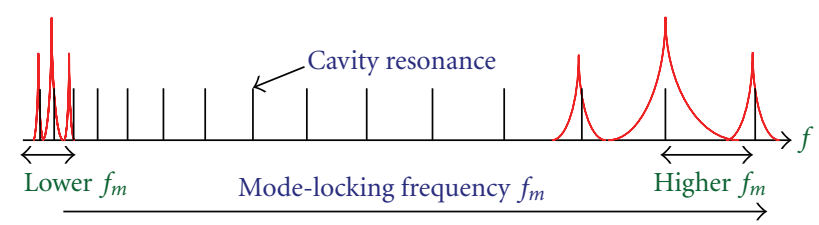

Figure 3: Principle of dispersion tuning: unevenly spaced resonance modes in the dispersive laser cavity.

modes in the dispersive laser cavity. Supposing that we apply a modulation at $f_{m}$ to the dispersive cavity strongly enough to be mode locked, the laser is obliged to operate at a frequency to meet the mode-locking condition. In the case of Figure 3 where $\beta_{2}$ is negative, lower $f_{m}$ stimulates lasing at lower frequency, and higher $f_{m}$ stimulates lasing at higher frequency. Reminding (7) and the harmonic mode locking of $N$ th order, the change of lasing frequency $\Delta \omega$ and the change of modulation frequency $\Delta f_{m}$ should have a relation,

$$
\Delta \omega=-\frac{\beta_{1}}{N \beta_{2} F_{0}} \Delta f_{m}=-\frac{\beta_{1}^{2} L}{N \beta_{2}} \Delta f_{m}=-\frac{n_{0}^{2} L}{c^{2} N \beta_{2}} \Delta f_{m} .
$$

Here we used $\beta_{1}=n_{0} / c$ and $n_{0}$ is the refractive index at $\omega=\omega_{0}$. It is sometimes more useful to rewrite (8) in terms of change of lasing wavelength $\Delta \lambda$. Using relations $\Delta \omega=$ $-2 \pi c \Delta \lambda / \lambda^{2}, \beta_{2}=-\lambda^{2} D / 2 \pi c$, where $D$ is the dispersion parameter, (8) can be transformed to

$$
\Delta \lambda=-\frac{n_{0}^{2} L}{c^{2} N D} \Delta f_{m}=-\frac{n_{0}}{c N D F_{0}} \Delta f_{m}=-\frac{n_{0}}{c D f_{m 0}} \Delta f_{m},
$$

where $f_{m 0}=N F_{0}$, which is the initial modulation frequency to give $\omega=\omega_{0}$. Thus the lasing frequency or wavelength can be tuned by changing the modulation frequency. This is called the dispersion tuning technique $[10,11]$. It is found from (9) that the wavelength shift is more sensitive to the change of modulation frequency when $D$ and $f_{m 0}$ are small, which means that the wavelength is not well defined and dispersion tuning is weak.

Wavelength sweep range $\Delta \lambda_{t r}$ is determined by two factors. One is the gain bandwidth, and the other is lasing at the adjacent harmonic mode, $(N-1)$ th or $(N+1)$ th mode. It happens when the change of modulation exceeds one FSR. From (9), $\Delta \lambda_{t r}$ is expressed as

$$
\Delta \lambda_{t r}=\frac{n}{c|D| N}=\frac{1}{|D| L f_{m 0}},
$$

However, (10) means that smaller $D, L$, and $f_{m 0}$ give wider sweep range.

The sweep speed $V_{s w}$ is inversely proportional to the photon lifetime as is the case of conventional swept lasers, that is, the sweep speed is faster when $L$ is small:

$$
V_{s w} \propto \frac{1}{L} .
$$

Since the laser is mode locked, the output is not in CW, but is pulsed at the repetition rate equal to the modulation 


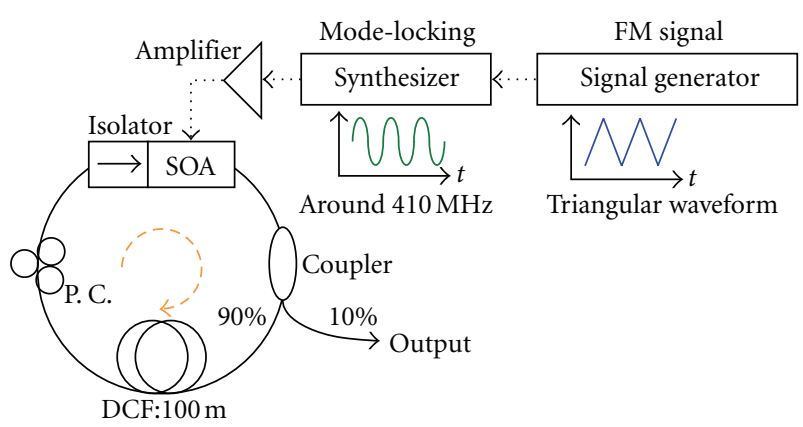

FIgURE 4: Dispersion-tuned swept fiber ring laser using SOA.

frequency. The pulse is strongly chirped and wide by the intracavity dispersion. The pulse from the mode-locked fiber laser with the intracavity dispersion is expressed with a chirped Gaussian pulse [11], whose spectral half width $\delta \omega$ is given by

$$
\delta \omega=\left(\pi \frac{f_{m 0}}{\lambda}\right)^{1 / 2}\left(\frac{8 \pi c M}{|D| L}\right)^{1 / 4}
$$

where $M$ is the modulation depth. This equation means that linewidth can be narrower as $f_{m 0}$ is smaller and $D$ and $L$ are larger. However, from (9), smaller $f_{m 0}$ increases the instability of lasing wavelength and causes linewidth broadening, which is the most cases in the following experiments. Thus $f_{m 0}$ should be as high as the linewidth broadening by (12) is acceptable.

From the above discussions, in order to realize wide and fast tuning with narrow spectral linewidth, $D$ and $f_{m 0}$ should be as large as possible, and $L$ should be as small as possible.

\section{Swept Fiber Ring Laser Using SOA at $1550 \mathrm{~nm}$ and $1300 \mathrm{~nm}$ Wavelength Bands}

Figure 4 shows a schematic construction of the dispersion tuning-based wavelength-swept fiber ring laser at the $1550 \mathrm{~nm}$ wavelength band. The laser is in a ring-laser configuration, and all the devices are pigtailed with singlemode fibers. The polarization-independent SOA module having a $3 \mathrm{~dB}$ gain bandwidth of $79.7 \mathrm{~nm}$ is used as the gain medium of the laser. Active mode-locking is realized by directly modulating the injection current to the SOA with the RF signal from an RF synthesizer. It can reduce the intracavity loss and the cost of external modulator. The triangular signal from a signal generator is input to the RF synthesizer for modulation frequency sweep. In order to provide the chromatic dispersion in the laser cavity needed for the dispersion tuning, we insert a 100m-long dispersion compensating fiber (DCF) having a dispersion parameter of $-90 \mathrm{ps} / \mathrm{nm} / \mathrm{km}$ at $\lambda=1550 \mathrm{~nm}$. An isolator in the SOA module ensures unidirectional lasing of the laser cavity. However, $10 \%$ of the light in the laser cavity is output from the 9:1 coupler.

We set the mode-locking frequency at around $410 \mathrm{MHz}$. It is determined by the RF modulation characteristics of

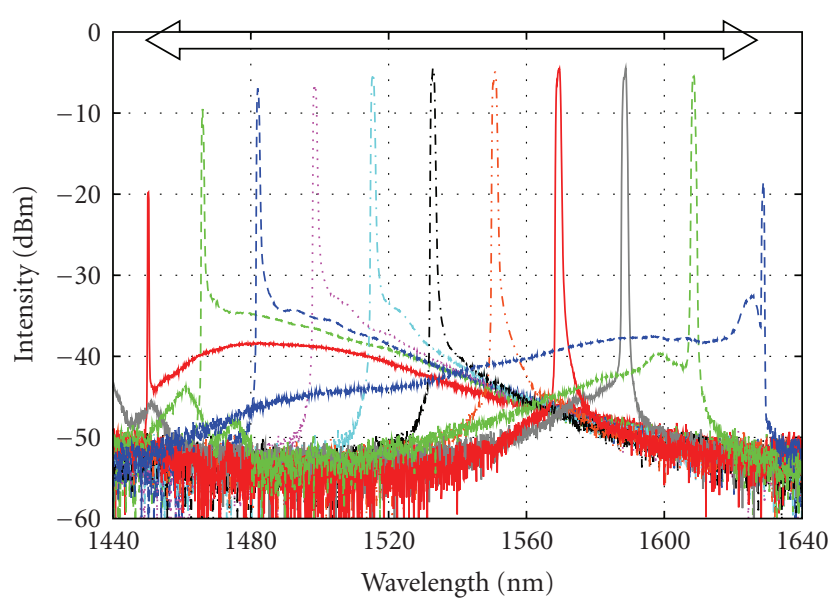

(a)

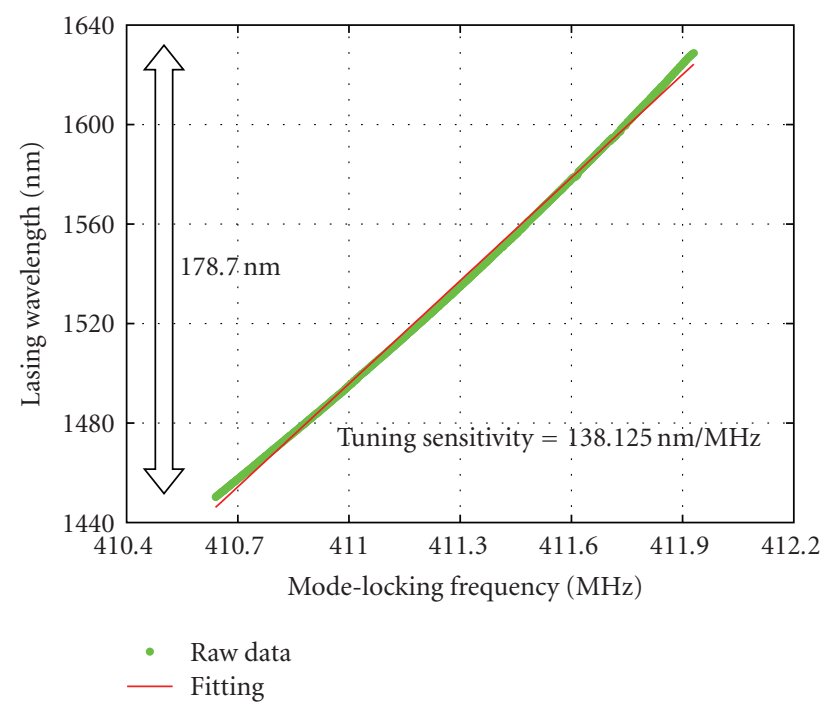

(b)

Figure 5: Static tuning characteristics at $1550 \mathrm{~nm}$ wavelength band: (a) lasing spectra (b) lasing wavelength again modulation frequency.

the SOA. Figure 5 shows the change of the lasing spectra and the lasing wavelength, respectively, as the mode-locking frequency is changed manually. The results suggest that the lasing wavelength almost shifts linearly toward the longer wavelength as the mode-locking frequency increases. The tuning sensitivity is $138.12 \mathrm{~nm} / \mathrm{MHz}$ and the static tuning range is as wide as $178.7 \mathrm{~nm}$. The output power is $\sim 1.3 \mathrm{dBm}$ and the instantaneous linewidth is $\sim 1.1 \mathrm{~nm}$ when the lasing wavelength is $1540 \mathrm{~nm}$. The instantaneous linewidth is almost the same at any wavelengths. Figure 6 is the temporal waveform of the output pulses at the modulation frequency of $400 \mathrm{MHz}$. It is found that the pulse is very wide, $\sim 1 \mathrm{~ns}$, because it is strongly chirped by the intracavity dispersion.

The mode-locking frequency at around $410 \mathrm{MHz}$ needs to be linearly modulated with the triangular waveform which is schematically shown in Figure 4 so as to sweep the wavelength linearly. The triangular waveform has two scan 


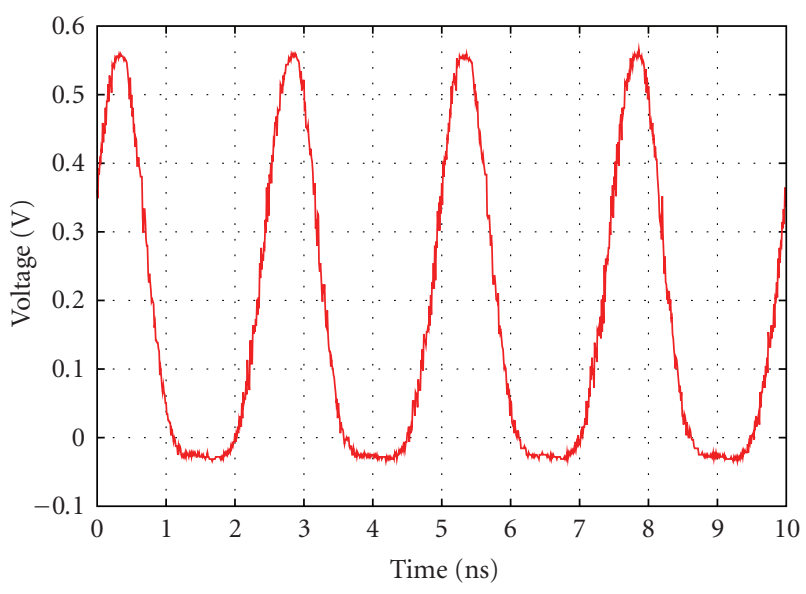

FIGURE 6: Temporal waveform of the output pulses at the modulation frequency of $400 \mathrm{MHz}$.
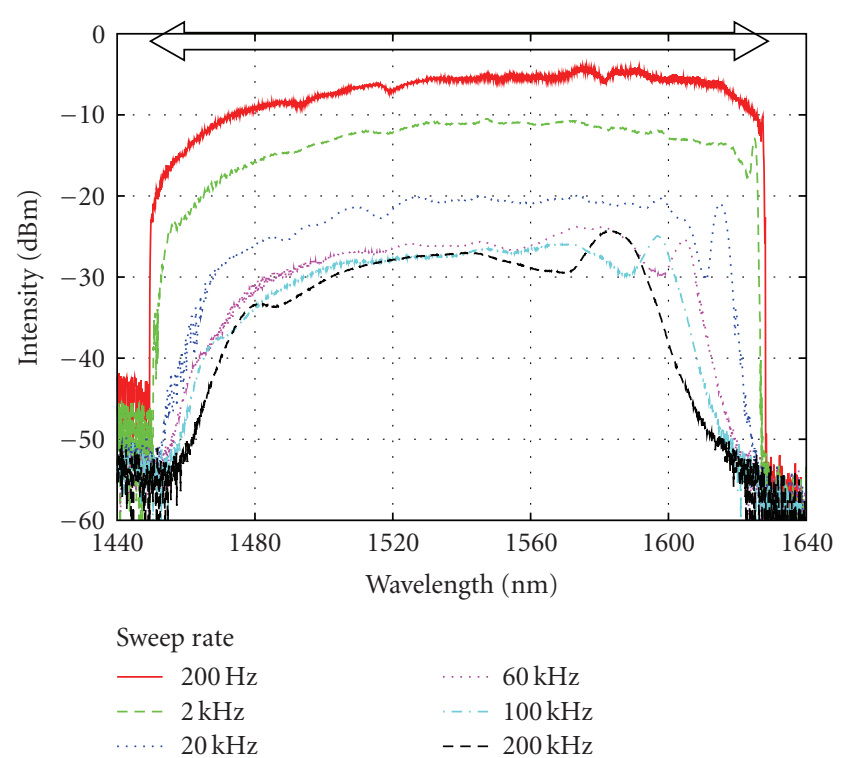

(a)

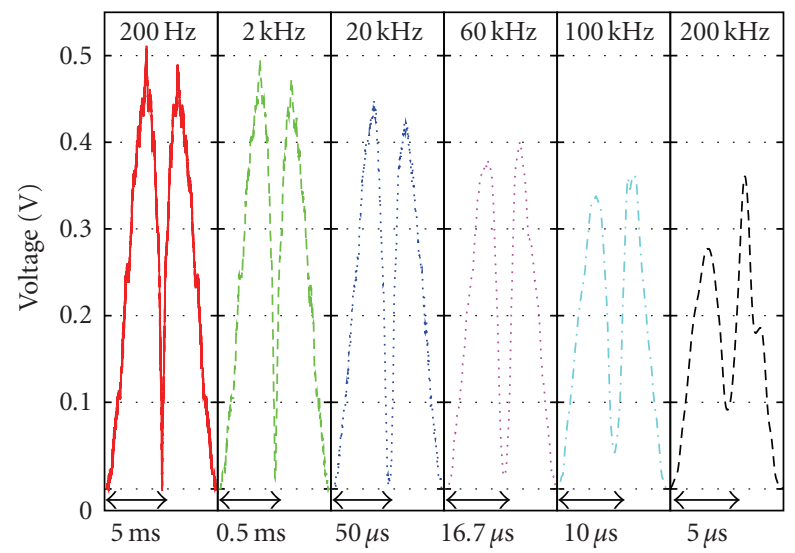

(b)

Figure 7: Dynamic sweep characteristics at $1550 \mathrm{~nm}$ wavelength band: (a) Max. hold spectra (b) temporal waveforms at different scan rates.

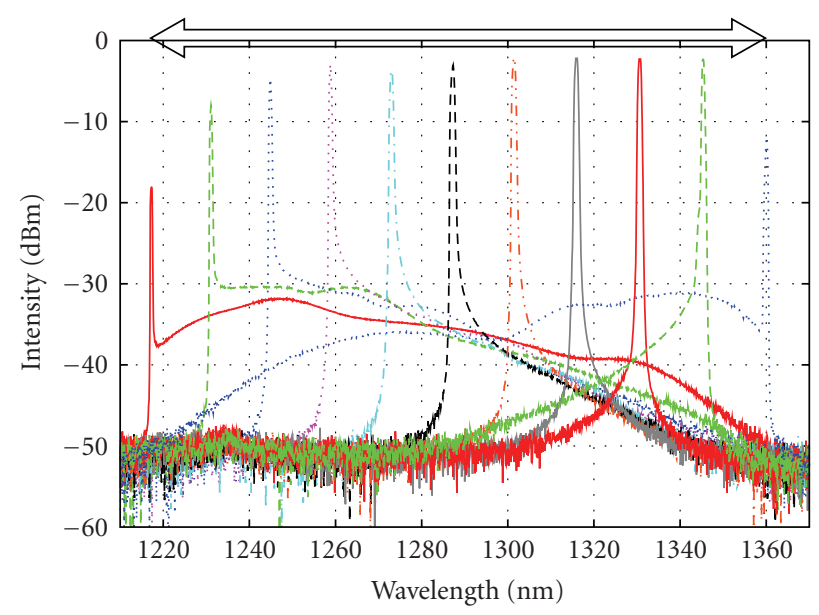

(a)

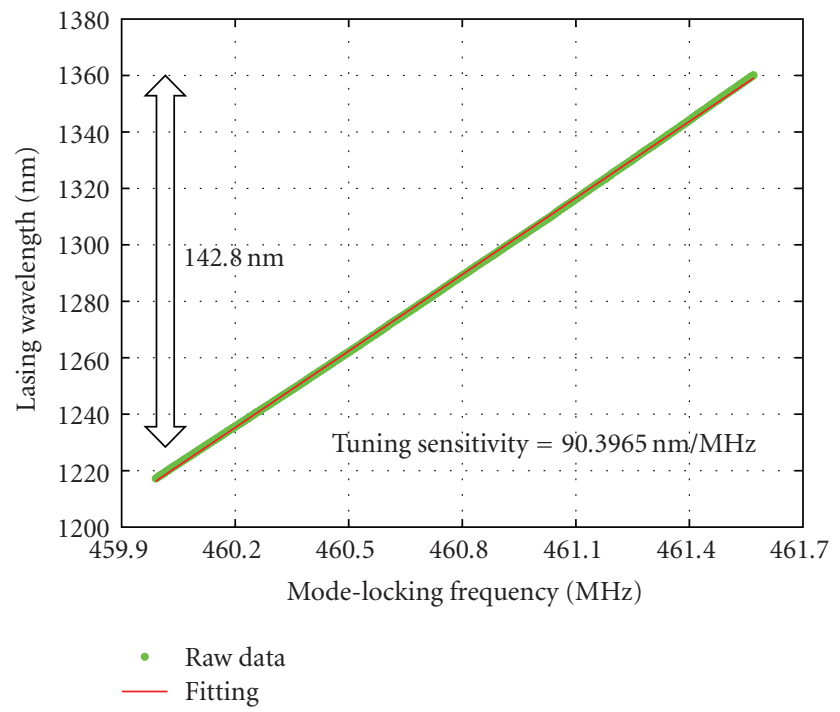

(b)

FIGURE 8: Static tuning characteristics at $1300 \mathrm{~nm}$ wavelength band: (a) lasing spectra (b) lasing wavelength again modulation frequency.

areas, upscan and downscan. In the upscan area, the lasing wavelength shifts-toward longer wavelength. Figure 7(a) shows the peak-hold spectra using a function of the optical spectrum analyzer, and Figure 7(b) shows the temporal waveforms when the scan rate is changed, $200 \mathrm{~Hz}, 2 \mathrm{kHz}$, $20 \mathrm{kHz}, 60 \mathrm{kHz}, 100 \mathrm{kHz}, 200 \mathrm{kHz}$. A dynamic sweep range of over $120 \mathrm{~nm}$ is achieved at scan rate over $100 \mathrm{kHz}$. The difference between the upscan and the downscan can be attributed to the nonlinear effect in SOA [12]. The output power is $-1.95 \mathrm{dBm}$ when this laser is swept at scan rate of $200 \mathrm{kHz}$.

As described earlier, our laser has wide and fast sweep characteristics. Applying this swept laser enables to allocate a large number of FBG array and a dynamic measurement in FBG sensing system. Currently, the tuning rate is determined only by the photon lifetime. Note that higher tuning rate 

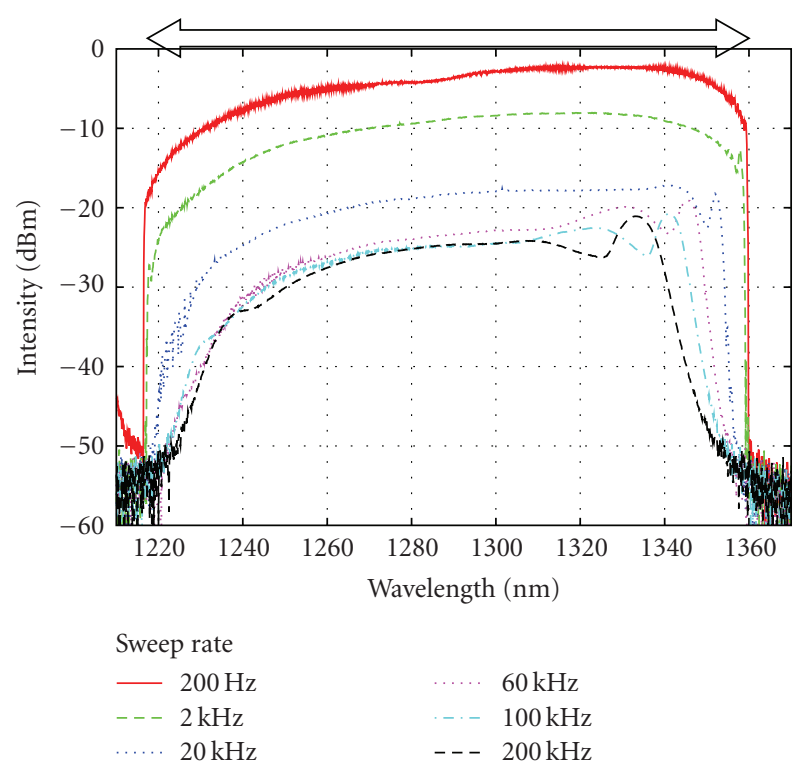

(a)

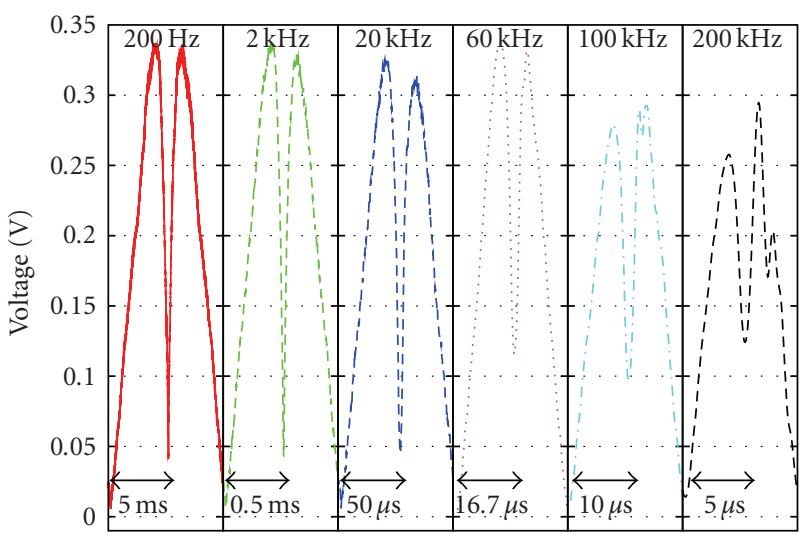

(b)

Figure 9: Dynamic sweep characteristics at $1300 \mathrm{~nm}$ wavelength band: (a) Max. hold spectra (b) temporal waveforms at different scan rates.

is possible by reducing the cavity length with use of higher dispersive element.

Operation wavelength band can be easily shifted by simply changing the gain medium in our swept fiber lasers. Here we show the swept fiber ring laser using SOA at $1300 \mathrm{~nm}$ wavelength band. The experimental setup is the same as in Figure 4, except that the SOA and other devices are replaced by similar devices designed for $1300 \mathrm{~nm}$. We used the same $100 \mathrm{~m}$-long DCF as the dispersive medium. The dispersion parameter of DCF is estimated to be around $-130 \mathrm{ps} / \mathrm{nm} / \mathrm{km}$ at $\lambda=1300 \mathrm{~nm}$. We chose the mode-locking frequency around $460 \mathrm{MHz}$.

Figure 8 shows the change of the lasing spectra and the lasing wavelength, respectively, as the mode-locking frequency is changed manually. The tuning range is as wide as $140 \mathrm{~nm}$. The output power is $\sim-0.78 \mathrm{dBm}$ and the instantaneous linewidth is $\sim 0.9 \mathrm{~nm}$ when the lasing wavelength is $1286 \mathrm{~nm}$. The instantaneous linewidth is almost the same

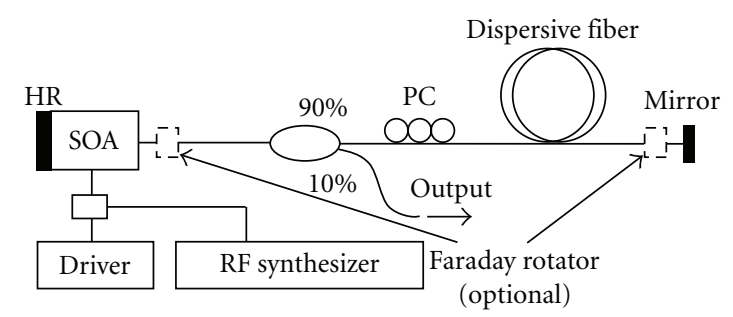

FIGURE 10: Dispersion-tuned swept fiber linear laser using SOA.

at any wavelengths. Figure 9(a) shows the peak-hold spectra when the modulation frequency is swept by the triangular waveform, and Figure 9(b) shows the temporal waveforms for several scan rates. A dynamic tuning range of over $120 \mathrm{~nm}$ is again achieved at scan rate over $100 \mathrm{kHz}$.

\section{Swept Fiber Linear Laser Using SOA at $1550 \mathrm{~nm}$ and $800 \mathrm{~nm}$ Wavelength Bands}

In the previous section, we demonstrated wide and fast wavelength-swept fiber lasers in the ring cavity configuration. The ring lasers, however, require an intracavity isolator to ensure the stable unidirectional lasing. In some wavelength bands, such as $800 \mathrm{~nm}$, isolators are not readily available. By contrast, linear cavity configuration does not require an intracavity isolator, therefore, is versatile in terms of operational wavelength. Here we show that the dispersion tuning is also applicable to the linear lasers, and demonstrate wide and fast wavelength-swept fiber linear laser using SOA at $1550 \mathrm{~nm}$ and $800 \mathrm{~nm}$ wavelength bands.

The experimental setup of the wide and fast wavelengthswept fiber linear lasers at $1550 \mathrm{~nm}$ wavelength band is shown in Figure 10. One end of an SOA is coated with a highly reflective (HR) mirror. Active mode-locking is also realized by directly modulating the injection current to the SOA with the RF signal from an RF synthesizer. The $300 \mathrm{~m}-$ long DCF, same with that of the ring lasers in the previous section, is inserted in the cavity. Another mirror is placed at the end of DCF. However 10\% of the light in the cavity is extracted from the 9:1 coupler as an output light. Since the SOA used in this experiment has polarization dependence, we used two Faraday rotators (FRs), one before the SOA, and the other before the end mirror to form a Faraday rotator mirror (FRM), in order to compensate the polarization fluctuation in the laser cavity [13]. There is no isolator in the cavity.

Figure 11 shows the change of the lasing spectra and the lasing wavelength, respectively, as the mode-locking frequency is adjusted manually. The tuning range is as wide as $110 \mathrm{~nm}$. The output power is $\sim 0.4 \mathrm{~d} \mathrm{Bm}$ and the instantaneous linewith is $\sim 0.7 \mathrm{~nm}$. Figure 12(a) shows the peak-hold spectra, and Figure 12(b) shows the temporal waveforms when the mode-locking frequency is modulated at a different scan rate. A dynamic sweep range of over $100 \mathrm{~nm}$ is again achieved at scan rate over $2 \mathrm{kHz}$. At higher scan rate, $20 \mathrm{kHz}$, the temporal waveform is degraded. This is because of the limit by the photon lifetime in the cavity. 


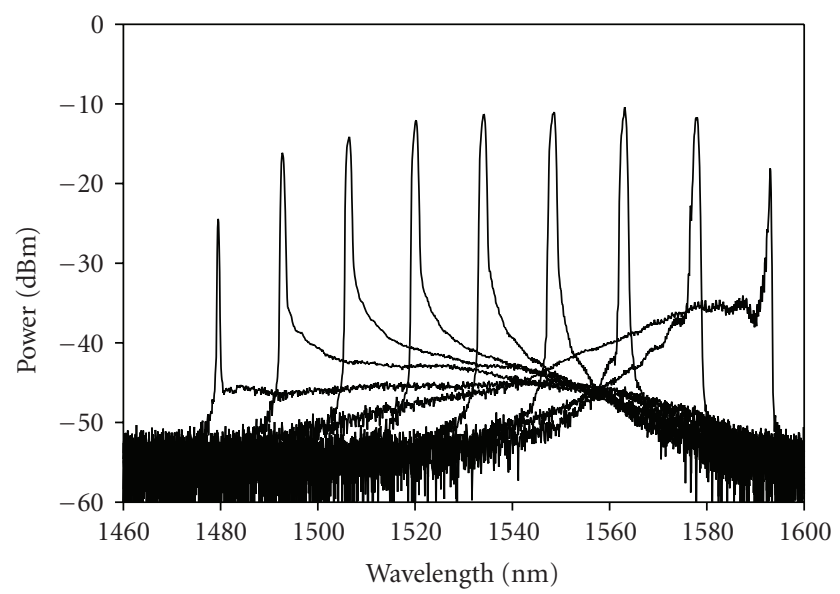

(a)

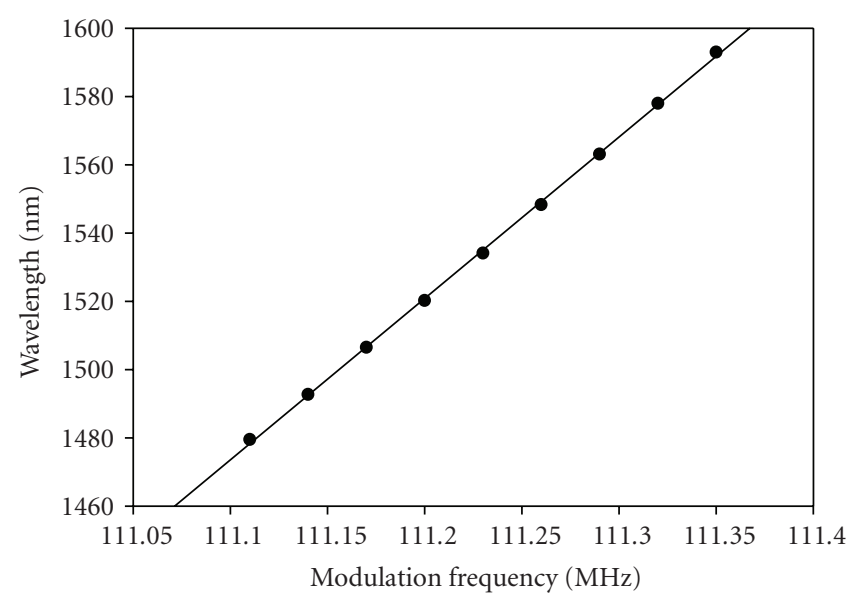

(b)

FIGURE 11: Static tuning characteristics at $1550 \mathrm{~nm}$ wavelength band: (a) lasing spectra (b) lasing wavelength again modulation frequency.

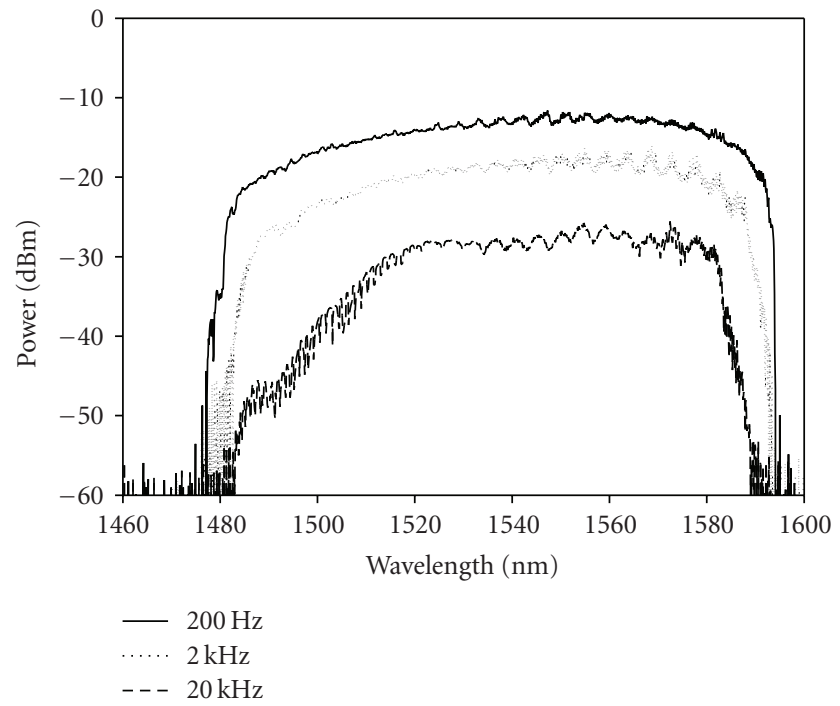

(a)

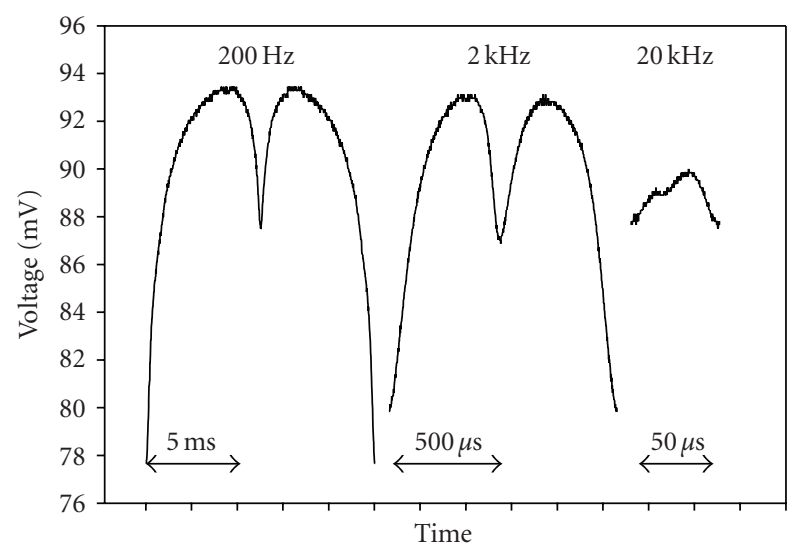

(b)

FIGURE 12: Dynamic sweep characteristics at $1550 \mathrm{~nm}$ wavelength band: (a) Max. hold spectra (b) temporal waveforms at different scan rates.
The round trip length in the linear cavity is twice as long as the cavity length, so it corresponds to the case of $600 \mathrm{~m}$-long ring laser. We expect that faster sweep will be possible with the shorter DCF length.

We also composed the wide and fast wavelength-swept fiber linear lasers at $800 \mathrm{~nm}$ wavelength band. The experimental setup is basically the same as in Figure 10, except that the SOA and other devices are replaced by similar devices designed for $800 \mathrm{~nm}$, and the FRs are not used since they are not available at this wavelength band. As for the dispersive fiber, we used a $300 \mathrm{~m}$-long single mode fiber for $800 \mathrm{~nm}$ wavelength band, which has large material dispersion $D$ $-100 \mathrm{ps} / \mathrm{nm} / \mathrm{km}$. Figure 13 shows the change of the lasing spectra and the lasing wavelength, respectively, as the change of mode-locking frequency. The tuning range is around $45 \mathrm{~nm}$, which is limited by the gain bandwidth of the SOA used in the experiment. The output power is $\sim-10 \mathrm{dBm}$ and the instantaneous linewith is $\sim 0.67 \mathrm{~nm}$. Figure 14 (a) shows the peak-hold spectra, and Figure 14(b) shows the temporal waveforms. A dynamic sweep range is again about $45 \mathrm{~nm}$, achieved at scan rate over $2 \mathrm{kHz}$. However, the peak-hold spectra are not flat, possibly due to the gain spectrum of the SOA and polarization change as the change of wavelength. Again, at higher scan rate, $20 \mathrm{kHz}$, the temporal waveform is degraded because of the long cavity length. We plan to address these issues in near future.

\section{Swept Fiber Ring Laser Using EDFA at $1550 \mathrm{~nm}$ Band}

So far, we used SOAs as the gain media, and the direct injection current modulation to the SOA was used for achieving mode locking needed for the dispersion tuning. Advantages of SOAs are the very wide gain bandwidth, and the capability of direct modulation. One drawback is the $\mathrm{RF}$ direct modulation characteristics in SOAs. As discussed in Section 2, we should raise the modulation frequency as high as possible for stable wide and fast sweep, preferably 


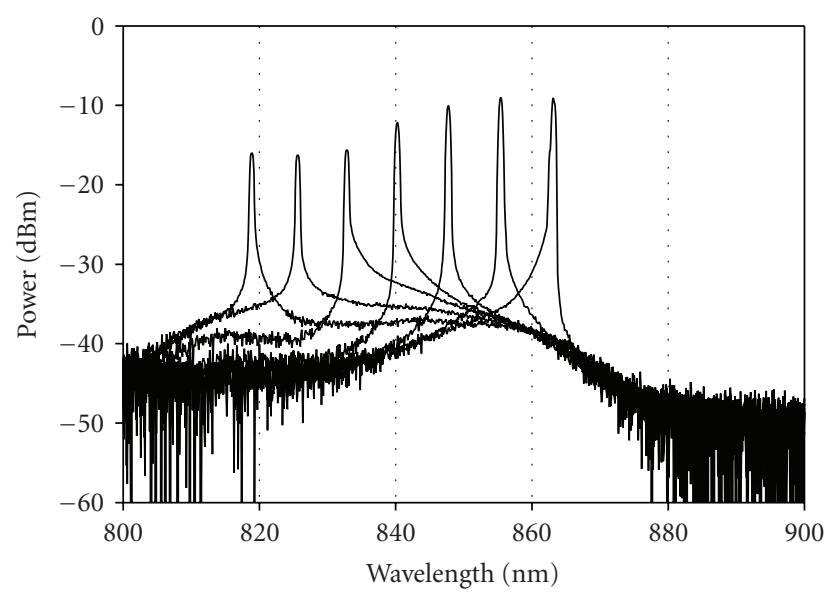

(a)

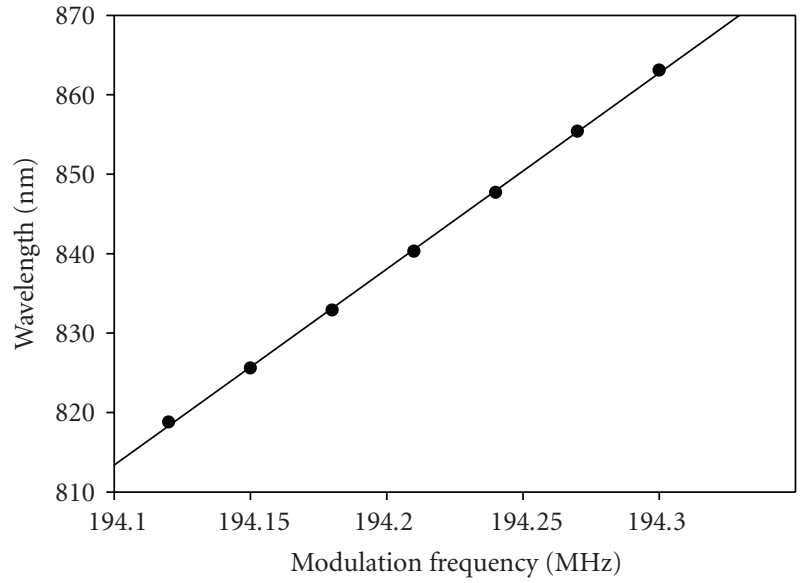

(b)

FIGURE 13: Static tuning characteristics at $800 \mathrm{~nm}$ wavelength band: (a) Lasing spectra (b) Lasing wavelength again modulation frequency.

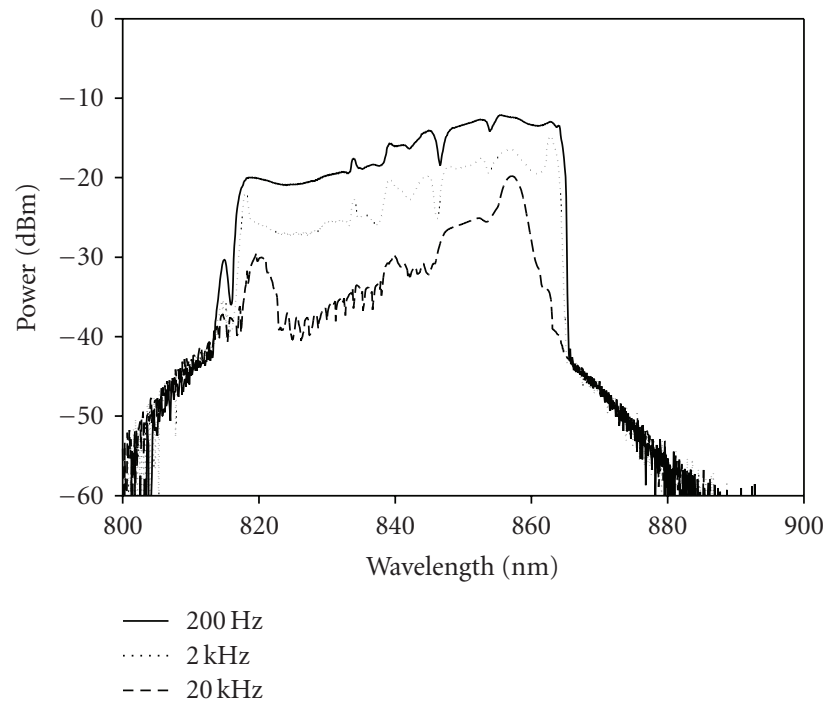

(a)

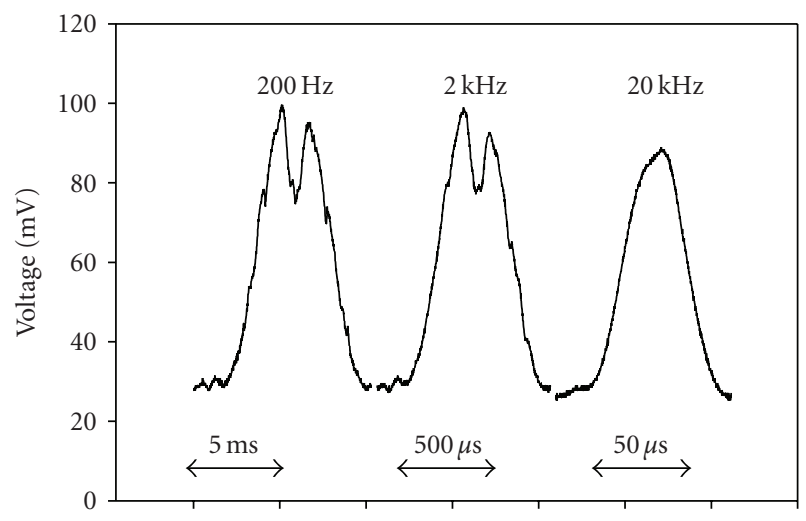

(b)

Figure 14: Dynamic sweep characteristics at $800 \mathrm{~nm}$ wavelength band: (a) Max. hold spectra (b) temporal waveforms at different scan rates.

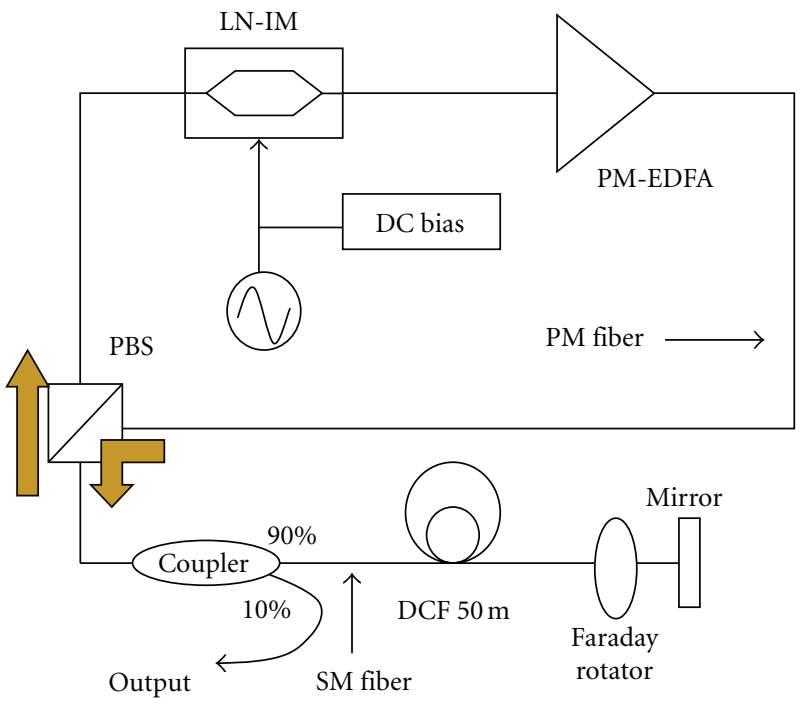

FIGURE 15: Dispersion-tuned swept fiber ring laser using EDFA.

higher than $1 \mathrm{GHz}$. However, the SOA chips and surrounding electronics in the module are not normally designed for accepting direct modulation at such high frequency. In the experiments using SOAs, we first measure the RF direct modulation characteristics of the SOA module, which are different one by one, and find the proper modulation frequency "sweet spot" for direct modulation. Another issue is the output power is not high, at best around $3 \mathrm{dBm}$.

In order to realize high-frequency modulation at high output power, we chose to use an external $\mathrm{LiNbO}_{3}$ intensity modulator as the mode locker, and an EDFA as the gain medium. The experimental setup is shown in Figure 15. Since the intensity modulator has strong polarization dependence, we had to compose the polarization maintaining (PM) type devices, including the EDFA (PM-EDFA). Since we do not have the PM-DCF, we chose to use so-called sigmalaser configuration using a polarization beam splitter (PBS) 


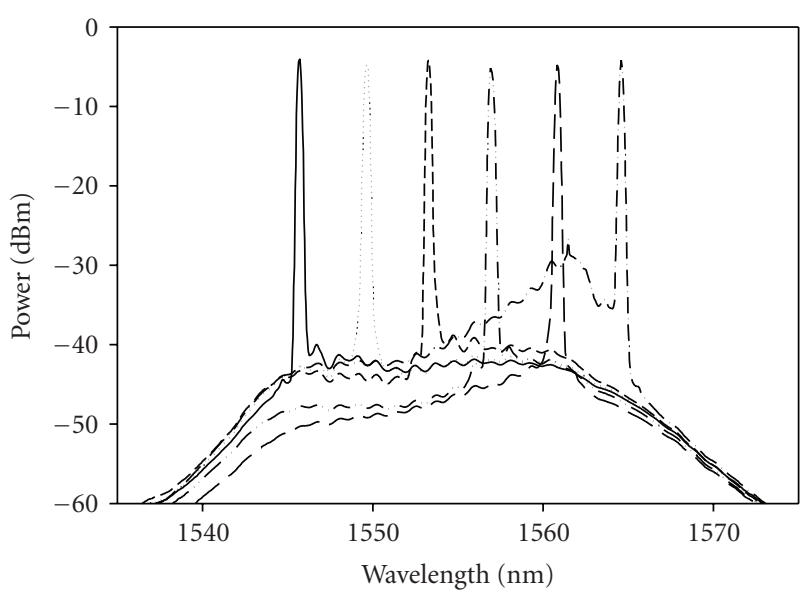

(a)

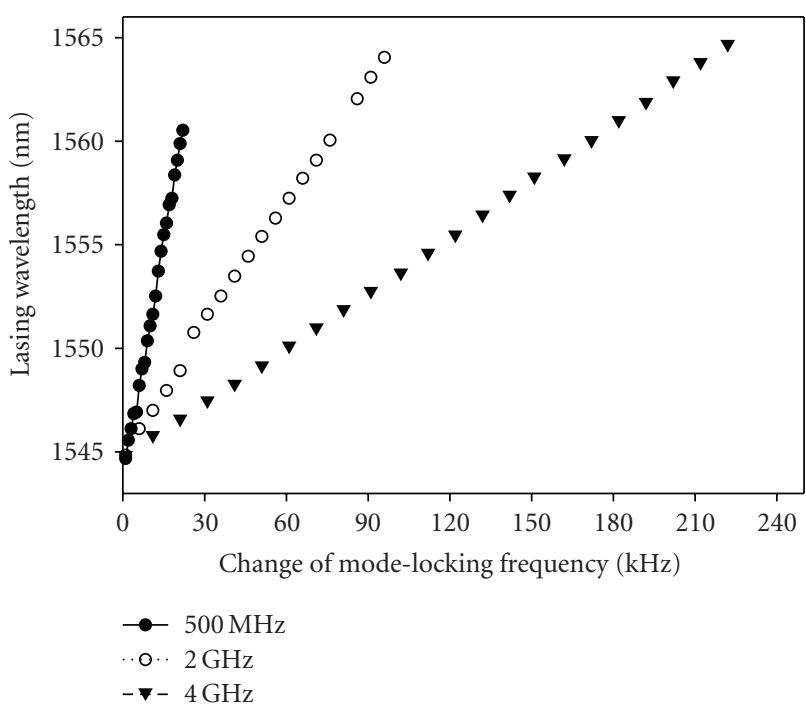

(b)

FIGURE 16: Static tuning characteristics: (a) lasing spectra (b) lasing wavelength again modulation frequency.

and an FRM, and the 50m-long DCF, same with the previous experiments, is placed in between. As the result, the intracavity polarization state is constant over entire wavelength band. Active mode-locking is realized by applying the intensity modulation by the intensity modulator. However, $10 \%$ of the light in the cavity is extracted from the 9:1 coupler as an output light.

Figure 16 shows the change of the lasing spectra and the lasing wavelength, respectively, as the change of modelocking frequency. The tuning range is not large, around $20 \mathrm{~nm}$, probably due to smaller gain bandwidth of the EDFA and large intracavity loss by the intensity modulator. Since the modulation frequencies are set at arbitrary values, we changed the modulation frequency from $500 \mathrm{MHz}$ to $2 \mathrm{GHz}$. As shown in Figure 16(b), it is shown that the slope of the lasing wavelength against modulation frequency becomes gentler as the modulation frequency gets higher, and tuning

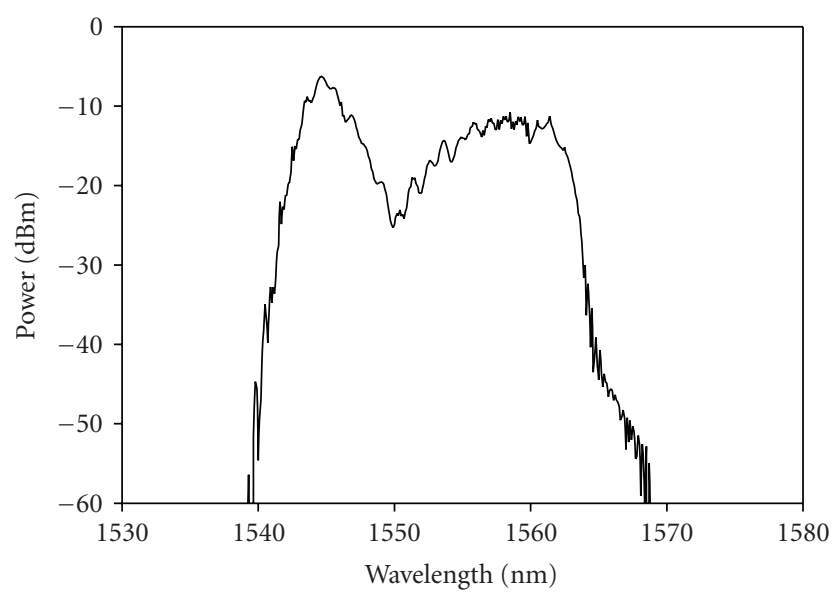

(a)

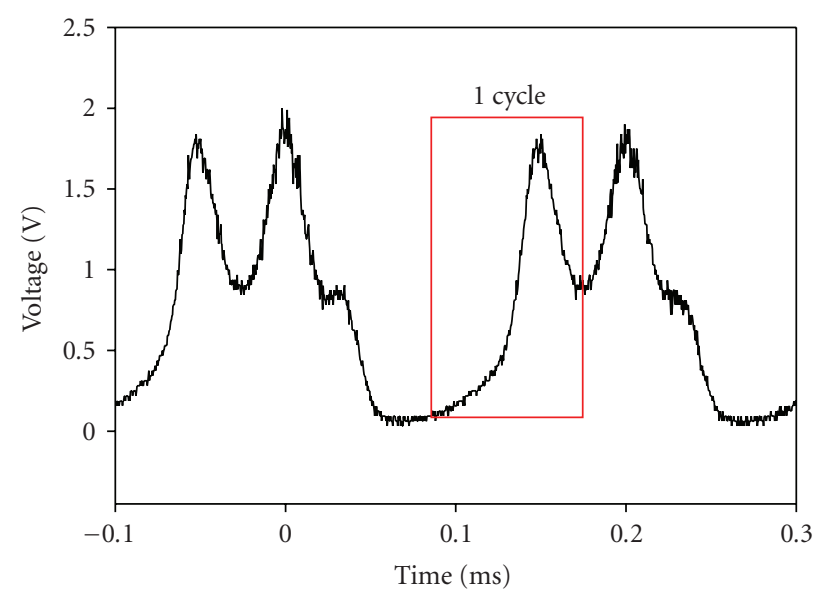

(b)

Figure 17: Dynamic sweep characteristics: (a) Max. hold spectra (b) temporal waveforms at different scan rates.

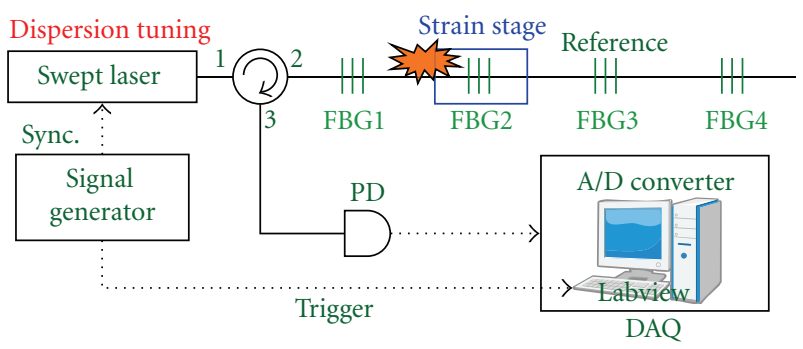

FIgURE 18: Experimental setup of dispersion-tuned swept-laser based FBG sensor at $1550 \mathrm{~nm}$ wavelength band.

range becomes wider, as expected from (9). Figure 17 shows the peak-hold spectra and the temporal waveforms when the modulation frequency is swept by the triangular waveform. A dynamic sweep range is again about $20 \mathrm{~nm}$, achieved at scan rate of $10 \mathrm{kHz}$. However, the peak-hold spectra are not flat, possibly due to the remaining polarization change in the cavity as the change of wavelength. 


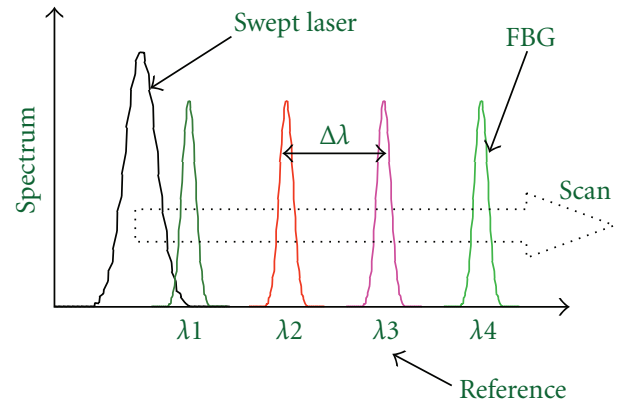

(a)

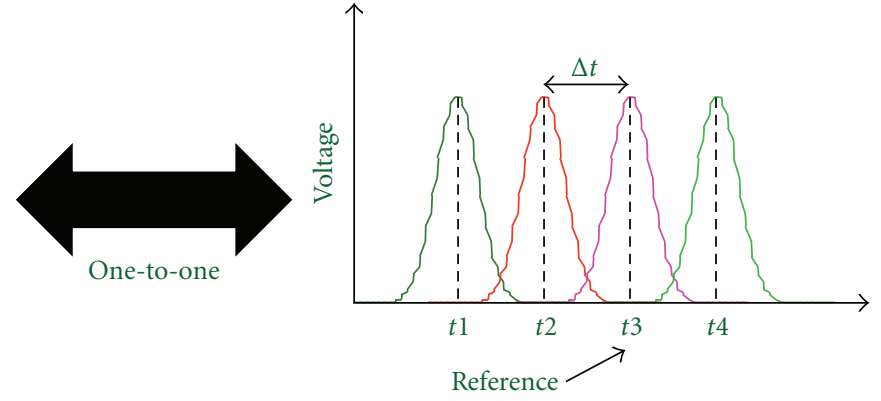

(b)

FIGURE 19: Sensing of FBG shift in the time domain using the swept laser.

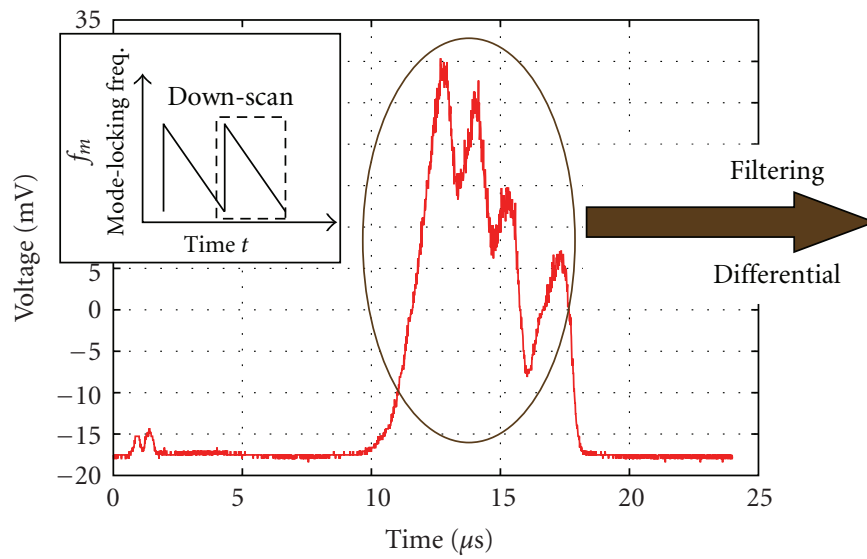

(a)

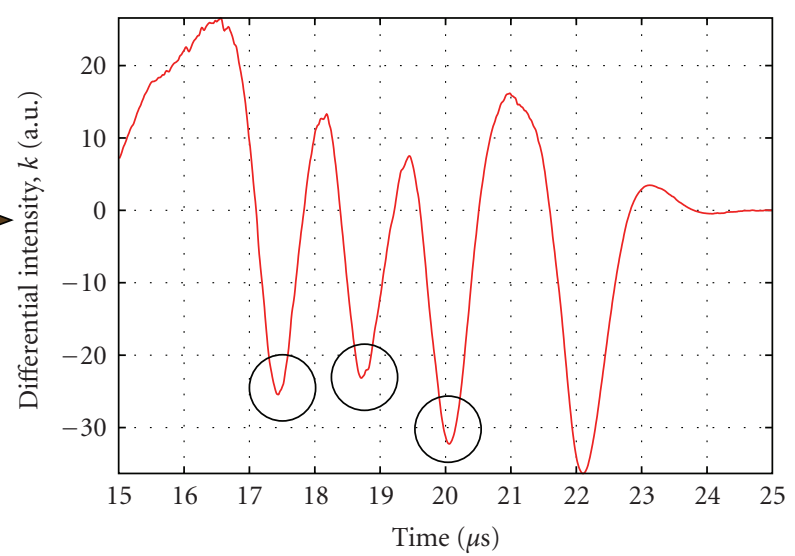

(b)

Figure 20: Temporal waveform, and its derivative waveform of the reflected light from the FBG array.

\section{Application to FBG Sensor}

The applications of the wavelength-swept laser are further investigated for an FBG sensing system. The experimental setup of our swept-laser based FBG sensor is shown in Figure 18. The swept laser is the SOA-based ring laser at $1550 \mathrm{~nm}$ wavelength band described in Section 3. The multiplexed FBG array consists of FBGs having different Bragg wavelengths, FBG1: $1525 \mathrm{~nm}, \mathrm{FBG} 2: 1540 \mathrm{~nm}, \mathrm{FBG} 3$ : $1550 \mathrm{~nm}$, FBG4: $1560 \mathrm{~nm}$, having more than 90\% reflectivity. The reflected light from the FBG array is led to a photodiode via a circulator. The trigger signal having the same frequency with the sweep signal is used to synchronize the $\mathrm{A} / \mathrm{D}$ converter. In order to control the $\mathrm{A} / \mathrm{D}$-converter and calculate the signals from FBGs, Labview is used. As shown in Figure 19(a), when the optical source is swept, the laser output light scans each FBG. Only the light corresponding to each FBG's Bragg wavelength is reflected and converted to an electrical signal by the photodiode as a sensor signal as shown in Figure 19(b). In this system, we can attribute each pulse in the temporal waveform to the corresponding FBG, and calculate the relative wavelength using the reference FBG. When the reference FBG is set to be FBG3 as shown in Figure 19, the relative wavelength $\Delta \lambda=|\lambda 2-\lambda 3|$ is estimated from $\Delta t=|t 2-t 3|$ by

$$
\Delta \lambda=\Delta t \cdot S_{s} \cdot \Delta \lambda_{t r}
$$

where $\Delta \lambda$ is the relative wavelength from the reference FBG, $\Delta t$ is the relative time from the reference FBG, $S_{s}$ is the sweep rate $(\mathrm{Hz})$ of the swept source, and $\Delta \lambda_{t r}$ is the sweep range $(\mathrm{nm})$ of the swept laser. Note that the $\Delta t(\Delta \lambda)$ changes in proportion to the strain added in FBG. In our experiment, in order to avoid errors due to the peak fluctuations in the temporal waveform, the waveform is filtered and differentiated. We track the minimal points in the differentiated waveform to calculate the relative wavelength.

Figure 20(a) shows the temporal waveform of the reflected light from the FBG array. Figure 20(b) is the sensor signal after filtering at $500 \mathrm{kHz}$ and differentiation when the tunable laser source is swept with $40 \mathrm{kHz}$ negative ramp waveform as shown in Figure 20(a). We chose to use only the downscan for interrogation of the FBG array, because the linearity of downscan is better than the upscan, as discussed in Section 3. Measurements at the scan rate of $40 \mathrm{kHz}$ are successfully achieved. Measured relative wavelengths from the reference FBG3 when no strain is applied to FBGs are summarized in Table 1. These results are obtained from 


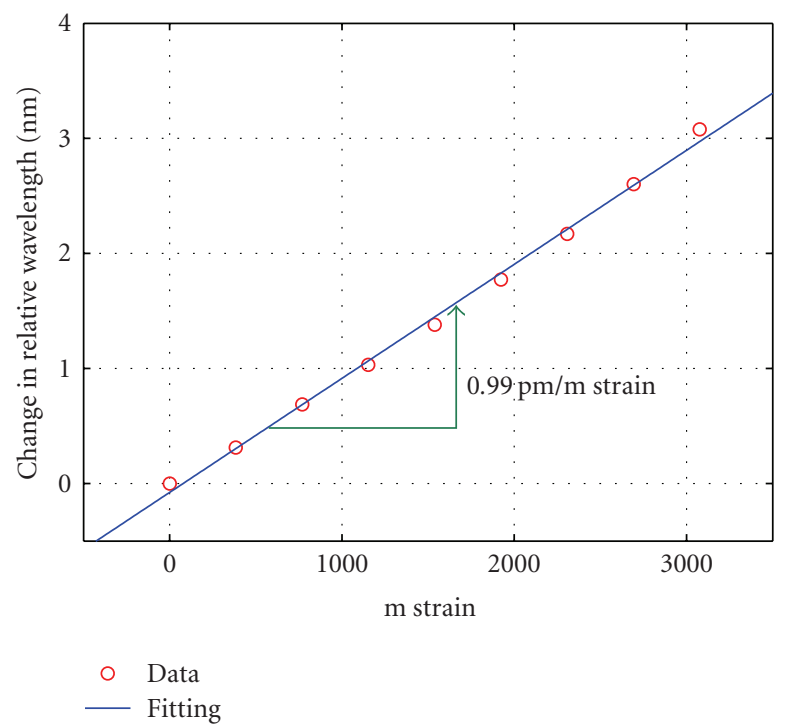

FIGURE 21: Wavelength shift of FBG2 against applied strain.

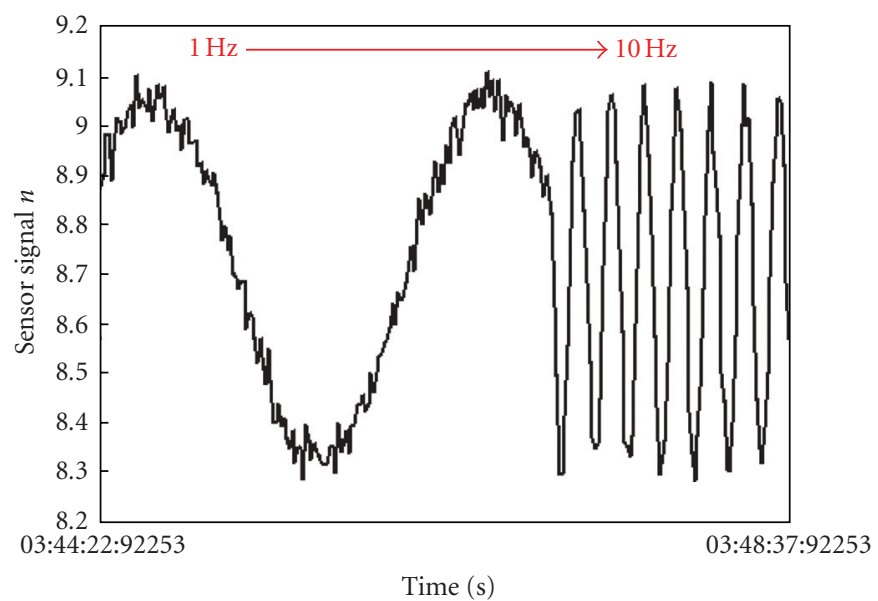

(a)

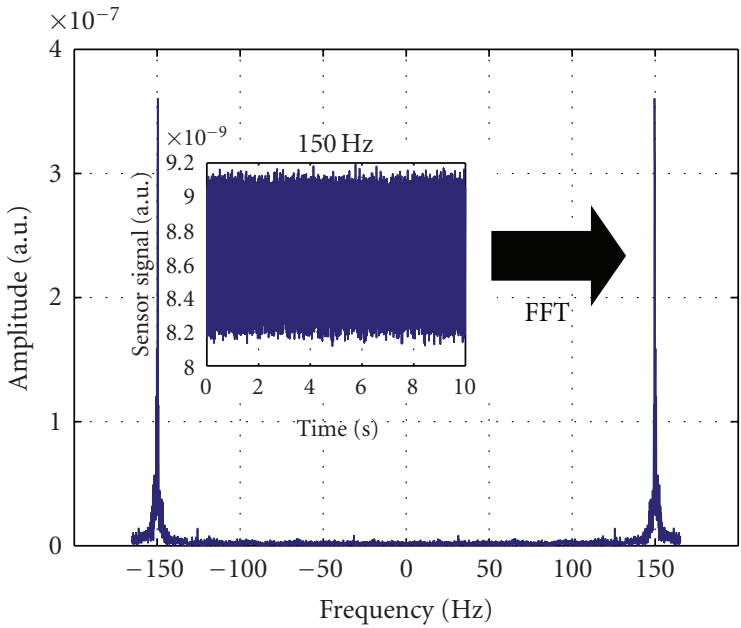

(b)

FIGURE 22: Vibration sensing results: (a) abrupt change from 1 to $10 \mathrm{~Hz}$ (b) $150 \mathrm{~Hz}$ waveform and its FFT data.

the data obtained for 5 seconds. Each average of relative wavelength from the reference FBG are almost equal to the Bragg wavelength of the FBGs used, and each standard deviation is also very small.

Figure 21 shows the change of relative wavelength when the strain is added to the FBG2 manually, in which the FBG is stretched by a steel slide manual stage. It is observed that the relative wavelength changes linearly as the strain is added. This result indicates that this sensor system works as expected. The slope of the linear-fitting is $0.99 \mathrm{~nm} /(\mathrm{m}$ strain).

Figure 22 shows the results of the dynamic sensing when periodical strain is added to the FBG2. The dynamic strain is applied by a PZT stage driven by a sinusoidal waveform from a RF function generator. When the frequency of the strain changes from 1 to $10 \mathrm{~Hz}$, it is confirmed that this sensor can capture the change of the frequency as shown in Figure 22(a). Figure 22(b) indicates that the dynamic strain can be measured correctly when the strain is added with a $150 \mathrm{~Hz}$ sinusoidal vibration. The FFT spectrum is calculated by MATLAB software. The resolution of this sensor is currently limited by the instantaneous linewidth of the swept laser since it is larger than the bandwidth of the FBGs, which will be able to be improved in the future. The results show that this system is capable to work accurately and measure transient distortion at a high measurement rate.

\section{Conclusion}

We reviewed our researches on the wide and fast wavelengthswept fiber lasers. We presented our proposed novel and different type of wide and fast wavelength-tunable fiber 
TABLE 1: Averages and standard deviations of the measured relative wavelengths when no strain is applied to FBGs.

\begin{tabular}{lcccc}
\hline Sensor results $\backslash$ FBG no. & $1(\mathrm{~m})$ & $2(\mathrm{~m})$ & $3(\mathrm{~m})$ & $4(\mathrm{~m})$ \\
Average & $23.91 \mathrm{n}$ & $9.20 \mathrm{n}$ & 0 & $9.40 \mathrm{n}$ \\
Standard Deviation & $35.37 \mathrm{p}$ & $28.10 \mathrm{p}$ & & $28.93 \mathrm{p}$ \\
\hline
\end{tabular}

laser. It uses the same gain medium with the conventional swept fiber lasers, but does not use any tunable filters. The tuning mechanism of our swept laser is dispersion tuning technique, which simply modulates the loss/gain in the dispersive laser cavity. By using wideband SOAs, the sweep range could be as wide as $\sim 180 \mathrm{~nm}$. Since the cavity contains no mechanical components, such as tunable filters, we could achieve the very high sweep rate, as high as $\sim 200 \mathrm{kHz}$. We have realized the swept lasers at three wavelength bands, $1550 \mathrm{~nm}, 1300 \mathrm{~nm}$ and $800 \mathrm{~nm}$, using SOAs along with EDFAs, and in two laser configurations, ring and linear ones. We also succeeded in applying the swept laser for dynamic FBG sensors.

For the further improvement of the scan speed of our laser, the cavity length has to be shorter, and the modulation frequency has to be higher. Our current choice of the dispersion element is the DCF, which satisfies both large dispersion, wide bandwidth, and low loss. Still we need higher dispersion with shorter length. Possible choices might be chirped FBG having very wide band, or photonic crystal fiber designed to have very high dispersion. Short cavity length is also desirable for enhancing the stability of the laser. As for the modulation frequency, currently it is limited by the RF modulation characteristics of the SOA module, as discussed in Section 5, so we expect that SOA modules designed for direct modulation are commercially available. For the further improvement of the sweep range of our laser, wideband SOA is necessary.

The sweep range is determined by two factors, the gain bandwidth and lasing at the adjacent harmonic mode, as discussed in Section 2. The latter factor can be avoided by the proper laser design, so the former factor is the main limiting factor. In the very wide swept laser, another problem will arise that the higher-order dispersion cannot be neglected, and as a result, the change of the lasing wavelength (or frequency) is not in linearly proportional to the change of the wavelength, whose indication has already been observed in Figure 5(b).

The instantaneous linewidth of the swept laser is currently around $1 \mathrm{~nm}$, much wider than the value calculated from (12) $(\sim 0.1 \mathrm{~nm})$. Thus, as discussed in Section 2, it should be improved by enhancing the modulation frequency. Actually, we have achieved the instantaneous linewidth around $0.2 \mathrm{~nm}$ with the modulation frequency of $1 \mathrm{GHz}$ in the first experiment [8].

We succeeded in applying the swept laser for dynamic FBG sensors. Our system is much better in terms of scan speed and sweep range than the existing FBG sensor systems, but its resolution is not yet as good as theirs, mainly due to the large instantaneous linewidth of the swept laser, which has to be improved in future.

\section{Acknowledgment}

This work was supported by Grant-in-Aid for Scientific Research (KAKENHI) B 18360163.

\section{References}

[1] A. D. Kersey, M. A. Davis, H. J. Patrick, et al., "Fiber grating sensors," Journal of Lightwave Technology, vol. 15, no. 8, pp. 1442-1462, 1997.

[2] D. C. Adler, Y. Chen, R. Huber, J. Schmitt, J. Connolly, and J. G. Fujimoto, "Three-dimensional endomicroscopy using optical coherence tomography," Nature Photonics, vol. 1, no. 12, pp. 709-716, 2007.

[3] M.-C. Amann and J. Buus, Tunable Laser Diodes, Artech House, Norwood, Mass, USA, 1998.

[4] S. Yamashita and M. Nishihara, "Widely tunable erbiumdoped fiber ring laser covering both C-band and L-band," IEEE Journal on Selected Topics in Quantum Electronics, vol. 7, no. 1, pp. 41-43, 2001.

[5] R. Huber, M. Wojtkowski, K. Taira, J. G. Fujimoto, and K. Hsu, "Amplified, frequency swept lasers for frequency domain reflectometry and OCT imaging: design and scaling principles," Optics Express, vol. 13, no. 9, pp. 3513-3528, 2005.

[6] S. H. Yun, C. Boudoux, G. J. Tearney, and B. E. Bouma, "Highspeed wavelength-swept semiconductor laser with a polygonscanner-based wavelength filter," Optics Letters, vol. 28, no. 20, pp. 1981-1983, 2003.

[7] R. Huber, M. Wojtkowski, and J. G. Fujimoto, "Fourier Domain Mode Locking (FDML): a new laser operating regime and applications for optical coherence tomography," Optics Express, vol. 14, no. 8, pp. 3225-3237, 2006.

[8] S. Yamashita and M. Asano, "Wide and fast wavelengthtunable mode-locked fiber laser based on dispersion tuning," Optics Express, vol. 14, no. 20, pp. 9299-9306, 2006.

[9] G. P. Agrawal, Applications of Nonlinear Fiber Optics, Academic Press, San Diego, Calif, USA, 2001.

[10] S. Li and K. T. Chan, "Electrical wavelength tunable and multiwavelength actively mode-locked fiber ring laser," Applied Physics Letters, vol. 72, no. 16, pp. 1954-1956, 1998.

[11] K. Tamura and M. Nakazawa, "Dispersion-tuned harmonically mode-locked fiber ring laser for self-synchronization to an external clock," Optics Letters, vol. 21, no. 24, pp. 19841986, 1996.

[12] S. H. Yun, C. Boudoux, M. C. Pierce, J. F. de Boer, G. J. Tearney, and B. E. Bouma, "Extended-cavity semiconductor wavelength-swept laser for biomedical imaging," IEEE Photonics Technology Letters, vol. 16, no. 1, pp. 293-295, 2004.

[13] Y. Takushima, S. Yamashita, K. Kikuchi, and K. Hotate, "Polarization-stable and single-frequency fiber lasers," Journal of Lightwave Technology, vol. 16, no. 4, pp. 661-668, 1998. 

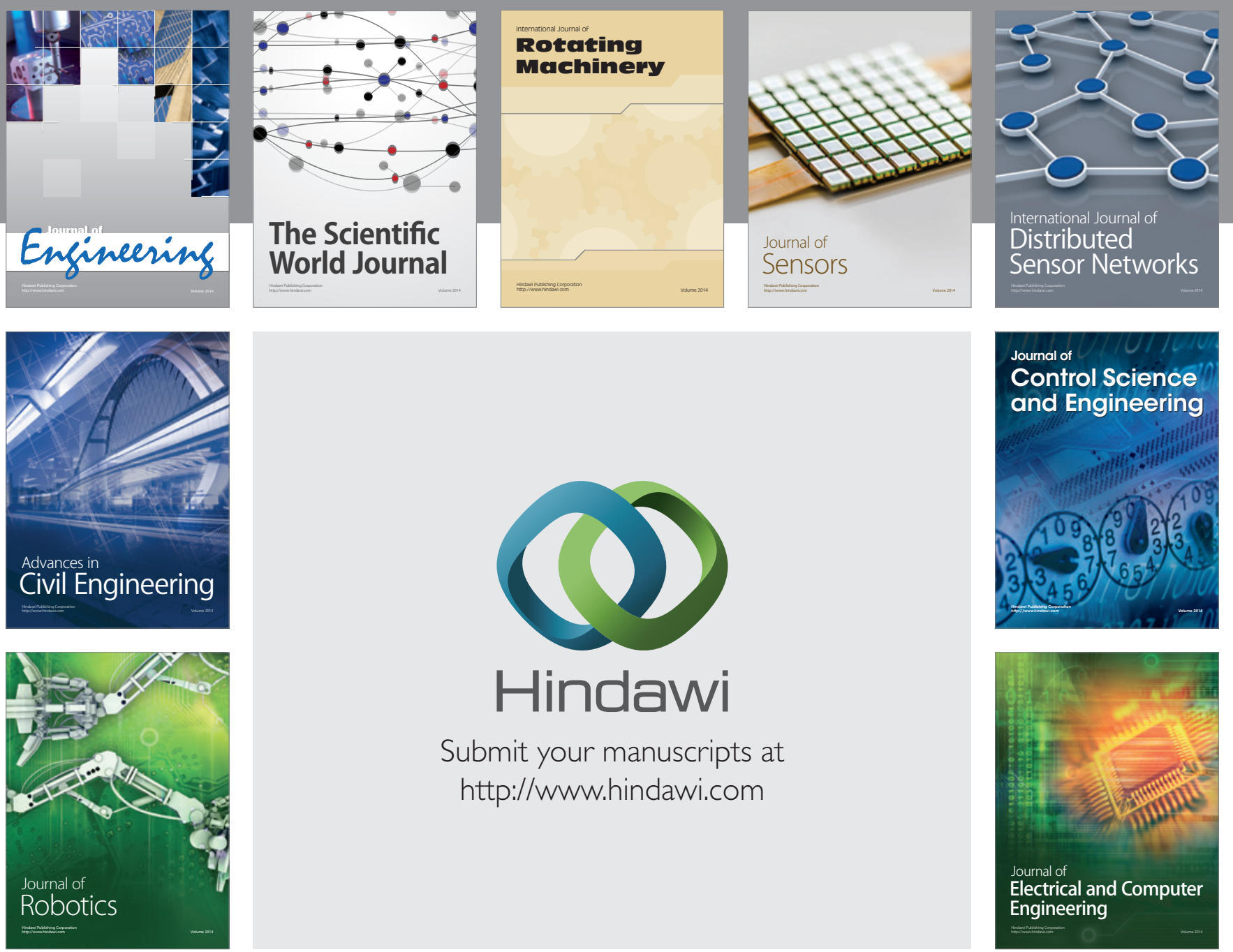

Submit your manuscripts at

http://www.hindawi.com
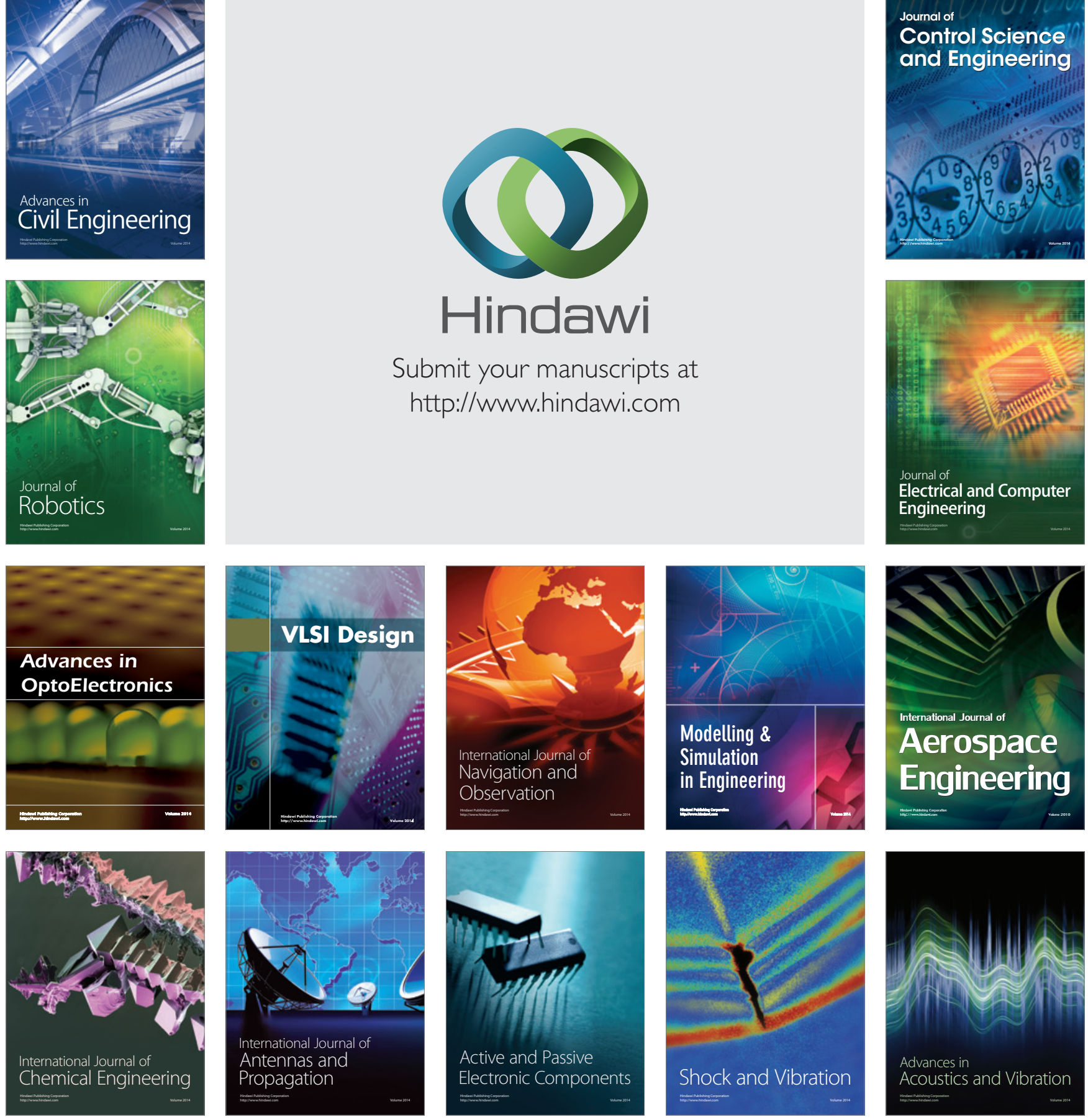